\title{
Multiparadigm Modeling of Dynamical Crack Propagation in Silicon Using a Reactive Force Field
}

\author{
Markus J. Buehler, ${ }^{1,2}$ Adri C. T. van Duin, ${ }^{2}$ and William A. Goddard III ${ }^{2, *}$ \\ ${ }^{1}$ Department of Civil and Environmental Engineering, Massachusetts Institute of Technology, 77 Massachusetts Avenue, Room 1-272, \\ Cambridge, Massachusetts 02139, USA \\ ${ }^{2}$ Division of Chemistry and Chemical Engineering, California Institute of Technology, 1200 E. California Boulevard, \\ Pasadena, California 91125, USA
}

(Received 17 August 2005; published 10 March 2006)

\begin{abstract}
We report a study of dynamic cracking in a silicon single crystal in which the ReaxFF reactive force field is used for several thousand atoms near the crack tip, while more than 100000 atoms are described with a nonreactive force field. ReaxFF is completely derived from quantum mechanical calculations of simple silicon systems without any empirical parameters. Our results reproduce experimental observations of fracture in silicon including changes in crack dynamics for different crack orientations.
\end{abstract}

PACS numbers: $62.20 . \mathrm{Mk}, 46.50 .+\mathrm{a}$

I. Introduction.-Brittle fracture is characterized by breaking of atomic bonds leading to formation of two new materials surfaces. Many existing atomistic models of fracture assume an empirical relationship between bond stretch and force. However, breaking of bonds in real materials is a complicated process that could previously only be captured with sufficient accuracy by quantum mechanical (QM) methods limited to approximately 100 atoms. Here we present a new multiscale simulation model derived from QM principles that is computationally efficient and capable of treating thousands of atoms with QM accuracy. This may open a new subdomain in materials modeling enabling a seamless integration of chemistry and mechanics.

Fracture of silicon has received tremendous attention due to its complexity of bond breaking and due to interesting failure dynamics observed experimentally [1-7]. Experimental efforts led to critical insight into deformation modes, such as crack instabilities and orientational dependence of crack dynamics in silicon single crystals [6].

Atomistic modeling fracture of silicon has been carried out using empirical force fields [8-11]. In contrast to metals, where fracture and deformation can often be described using the embedded atom method (EAM) [12-17], a proper description of fracture in silicon has proved to be far more difficult $[4,8,10,17-29]$. Early attempts to model fracture in Si used Tersoff's classical potential [30] (in the following referred to as "Tersoff potential") and similar formulations [31,32]. However, simulations carried out with those potentials did not reproduce experimentally observed brittle fracture of silicon [3]. It has been suggested that the reason for these discrepancies is that the description of the atomic bonding at large stretch obtained by empirical potentials deviates significantly from the quantum mechanical solution [3].

It is believed that in order to develop models of fracture of silicon that agree with experimental observations, the accuracy of QM for atoms near the propagating crack tip is necessary. This has been demonstrated in earlier work [9], where QM regions were coupled to empirical potentials and continuum regions. Because of computational limitations, the number of atoms treated with QM is limited to approximately 100 atoms. However, the zone near a crack tip in which bonds are stretched and broken often comprises of several thousand atoms.

Baskes and co-workers used their modified EAM (MEAM) to describe crack motion in silicon [10] to investigate the critical load for fracture initiation [11]. Even though this model leads to improved results compared to Tersoff-type potentials, the MEAM formulation cannot describe bond formation and breaking of silicon with other elements such as oxygen or charge flow.

We present an alternative approach based on the ReaxFF reactive force field developed to reproduce the barriers and structures for reactive processes from $\mathrm{QM}$, but at a computational cost many orders of magnitude smaller. We propose a hybrid simulation technique in which the ReaxFF reactive potential for silicon [33,34] is used for a modest region of a few thousand atoms close to the crack tip while a computationally inexpensive Tersoff potential [30] is used to describe more distant atoms needed to include their elastic constraints on the propagating tip. The Tersoff potential and ReaxFF lead to similar materials behavior at small strains, but deviate strongly at large strains [33]. The overlap at small strains enables a handshake between the two methods.

The ReaxFF reactive potentials $[33,34]$ have been developed to describe combinations of many different elements across the periodic table, including first row elements $(\mathrm{C}, \mathrm{O}, \mathrm{H}, \mathrm{N})$, metals $(\mathrm{Cu}, \mathrm{Al}, \mathrm{Mg}, \mathrm{Ni}, \mathrm{Pt})$, and semiconductors ( $\mathrm{Si}$ ) [33-38]. Thus, the methodology described here could potentially be a valuable tool for describing plasticity and fracture for materials where certain details of the bond breaking process are necessary to model crack propagation. Here we focus on modeling fracture in 
pure silicon and the interactions of silicon with oxygen competing with crack extension.

II. Hybrid atomistic modeling of cracking in silicon.The Computational Materials Design Facility (CMDF) is a Python [39] based framework [40,41]. Individual computational engines are wrapped using the simplified wrapper and interface generator for rapid integration of low-level codes with scripting languages [42]. CMDF enables complex multiscale simulation tasks encompassing a variety of simulation paradigms, such as the combination of ReaxFF with classical potentials such as Tersoff or EAM.

The ReaxFF potential for silicon [33,34] has been tested against QM for a wide range of processes [43], including $\mathrm{Si}-\mathrm{Si}$ bond breaking in $\mathrm{H}_{3} \mathrm{Si}-\mathrm{SiH}_{3}$ and $\mathrm{Si}=\mathrm{Si}$ bond breaking in $\mathrm{H}_{2} \mathrm{Si}=\mathrm{SiH}_{2}$, equations of state for 4-coordinate silicon (diamond configurations) and 6-coordinate silicon phases ( $\beta$ tin), and simple cubic crystal. It can also treat interactions of $\mathrm{Si}$ with $\mathrm{O}$ and $\mathrm{H}$ [34]. We added the stability and equation of state of a 5-coordinate Si-condensed phase to the Si training set, and reevaluated the $\mathrm{Si}-\mathrm{Si}$ bond and $\mathrm{Si}$ $\mathrm{Si}$-Si angle parameters to improve the fit to the energy and equation of state of this phase. All parameters are completely derived from QM calculations. Because of the complexity of the mathematical expressions describing the partial bond orders, energies, and charges [33], ReaxFF is 1 to 2 orders of magnitudes more expensive than the Tersoff potential, but several orders of magnitude faster than QM.

We use a simple approach to describe the transition region between two paradigms, described by two parameters, $R_{\text {trans }}$ for the size of the transition region, and $R_{\text {buf }}$ for the size of the ghost atom region (see Fig. 1). We model a reactive region $\Omega_{\mathbf{r x}}$ embedded into a nonreactive domain $\Omega_{\mathbf{n r}}$. The shape of the reactive region is updated every $N_{u}=10$ steps during the integration. We choose $R_{\text {trans }}=$ $6 \AA$ and $R_{\text {buf }}=5 \AA$. We have chosen these parameters by trial and error so that the crack dynamics is not affected by changing these parameters. The shape and size of the reactive region surrounding the moving crack is based on the strain energy density of each atom in the model. All atoms with a strain energy larger than $E_{\text {crit }}=-3.5 \mathrm{eV}$ are embedded in a cylindrical reactive region of $R=10 \AA$. This critical strain filters atoms at the tip of cracks and atoms in the vicinity of cracks whose bonds are stretched significantly. The union of all cylindrical regions yields the total reactive region, allowing representation of arbitrary shapes. The final reactive region is typically not circular, as can be clearly seen in Figs. 4 and 5. Initially, the systems contain several hundred reactive atoms corresponding to a small reactive region at the crack tip. This initial size of the reactive region may increase during the simulations approaching several thousand atoms because of crack branching or due to formation of microcracks.

When oxygen atoms are present in the system in addition to the criterion based on the strain energy density, each oxygen atom is embedded in a cylindrical reactive region of $R=10 \AA$.

The force and energy contribution from different simulation engines is weighted as shown in Fig. 1. Each computational engine $i$ has a weight $w_{i}$ associated with it. For each atom in the system, the weight of $N$ computational engines add up to unity $\sum_{i=0 \ldots N} w_{i}=1$. Forces and energies are weighted accordingly, and the force vector of an atom $j$ is calculated as $\vec{F}_{j}=\sum_{i=0 \ldots N} \vec{F}_{j, i} w_{i}$, where $\vec{F}_{j, i}$ is the force contribution on atom $j$ due to computational engine $i$ and $\vec{F}_{j}$ is the resulting force vector on atoms $j$.

Figure 2 depicts our atomistic model. We consider a crystal with an initial crack of length $a$. The thickness of the system is one unit cell in the $z$ direction with periodic boundary conditions, corresponding to a plane strain case $\left(L_{z}=5.43 \AA\right)$. This model is chosen to keep the reactive region small. We note that this choice of geometry may impose constraints on the system, which may effect the possibility of dislocation nucleation. We strain the slab with strain $\varepsilon_{x x}$ in mode I prior to simulation [24]. The boundaries are fixed so that the stress in the material can only be relieved by crack propagation.

III. Simulation results. - First we compare the dynamics of crack propagation described using pure Tersoff [30] to the results incorporating a region treated by ReaxFF at the crack tip to demonstrate the importance of QM accuracy at the crack tip. The crack direction is [110] with a (110) crack surface. We strain the system by $20 \%$ in mode I loading and then minimize the potential energy. We consider two cases, one in which a small region around the crack tip is treated using ReaxFF embedded in a Tersoff region, and the other case in which all atoms are described using Tersoff. The results of these computational experiments are shown in Fig. 3. We observe that with pure Tersoff, the crack does not propagate. Instead the crack becomes blunt [Fig. 3(a)] and eventually amorphizes as the loading is increased sufficiently. This incorrect behavior has been observed in previous studies with Tersoff-type potentials $[4,11]$, and is in sharp contrast to experimental results [6], suggesting brittle behavior of silicon, in particular, when initial cracks have (110) faces. In agreement with experiment, our hybrid treatment leads directly to a
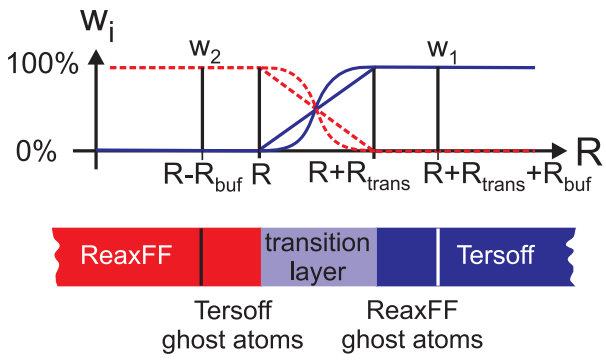

FIG. 1 (color online). The interpolation method for defining a mixed Hamiltonian in the transition region between two paradigms. As an alternative to the linear interpolation we have also implemented the smooth sinusoidal function. 


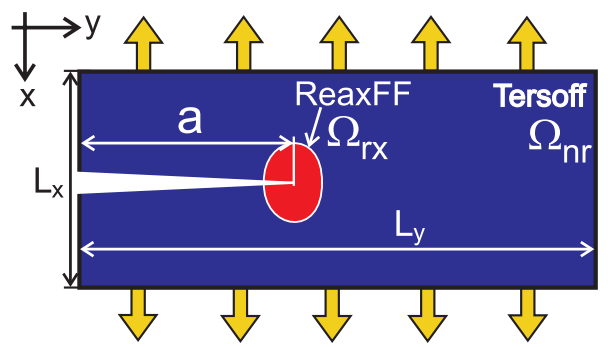

FIG. 2 (color online). Geometry used for simulating mode I fracture in silicon. The systems contain between 13000 and 113015 atoms with $L_{x} \approx 550 \AA$ and $L_{y} \approx 910 \AA$. We can treat up to 3000 atoms with ReaxFF in $\Omega_{\mathbf{r x}}$.

correct description of fracture [Fig. 3(b)] [1,3,4,6]. For the [100] crack direction with (100) crack surfaces, similar behavior is observed.

Figure 4(a) shows various snapshots of a crack propagating in a silicon crystal strained by $10 \%$ with the temperature controlled to be around $T \approx 300 \mathrm{~K}$. The crack propagates through the material in a perfectly brittle manner. The crack approaches a speed of $3.4 \mathrm{~km} / \mathrm{sec}$, which is about $75 \%$ of the Rayleigh-wave speed, the limiting speed predicted by continuum theory [44] $\left(c_{R, \mathrm{Si}} \approx 4.5 \mathrm{~km} / \mathrm{sec}\right.$ [45]). Figure 4(b) depicts the results for the same system but strained by $20 \%$. Here we observe an initial phase of mirrorlike cleavage, followed by a regime in which the crack leaves a rougher surface. These observations agree with experimental studies of cracks propagating along the same crystallographic planes [6]. Our results seem to resemble a universal behavior of dynamical cracks in homogeneous materials showing a mirror-mist-hackle transition, as has been confirmed experimentally $[6,46,47]$. Further, we observe that fracture dynamics in this case of large loading and elevated temperature leads to a mechanism of formation of secondary microcracks ahead of the primary crack [see, for example, Fig. 4(b)]. This microcracking mechanism is similar to the experimental observations [48] and are consistent with theoretical analyses [49].

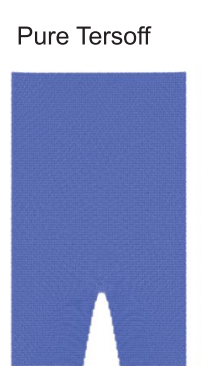

(a)

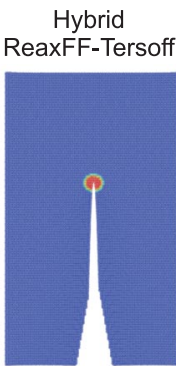

(b)

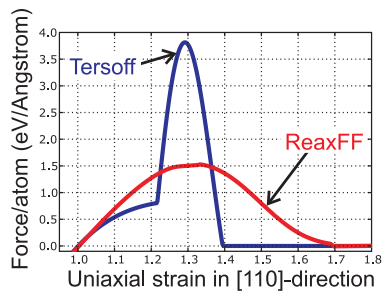

(c)
FIG. 3 (color online). Crack propagation with a pure Tersoff potential (a) and the hybrid ReaxFF-Tersoff model (b) along the [110] direction (energy minimization scheme). The blue regions are Tersoff atoms, the red regions are reactive atoms. The systems contain 28000 atoms with $L_{x} \approx 270 \AA \times L_{y} \approx 460 \AA$. Subplot (c) shows the difference in large-strain behavior between Tersoff and ReaxFF. Both descriptions coincide at small strain.
Figure 4(c) shows crack dynamics for an initial crack oriented into the [100] direction [4]. We observe that the crack branches off into two [110] directions-forming (110) crack surfaces - shortly after nucleation. This result is consistent with the notion that crack dynamics is most stable along this direction and agrees with experiment [4]. This is not observed with the pure Tersoff model.

The potential inherent in our hybrid scheme become apparent when reactive chemical processes compete with brittle crack extension. Figure 5 depicts studies in which we investigate crack dynamics in a small penny-shaped crack containing oxygen molecules $\mathrm{O}_{2}$. As previously, the slab is under mode I tensile loading. The oxygen atoms react strongly with the silicon surface [Fig. 5(b)]. We notice that $5 \%$ prestrain is sufficient to nucleate a crack for pure silicon, but when oxidative processes are present such a strain is not sufficient to nucleate a crack. The oxidation leads to crack blunting effectively reducing the stress intensity factor at the crack tip, and leads to formation of strong $\mathrm{Si}-\mathrm{O}$ bonds.

IV. Discussion and conclusion. -We have presented a new numerical method integrating the ReaxFF and Tersoff force fields to allow a physics based description of fracture of silicon. Our new scheme represents a multiscale approach coupling the QM scale of chemistry and bond breaking and formation with the scale of mechanics of materials. Unlike previous attempts, our method is completely based on first-principles, with no empirical parameters used.

(a)

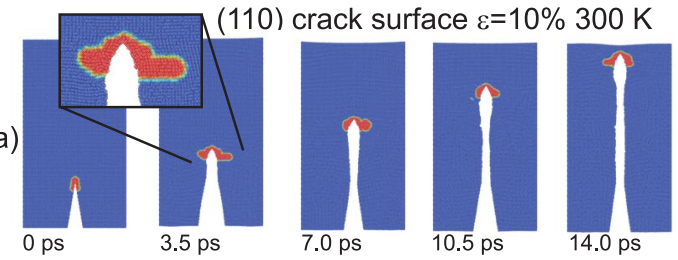

(110) crack surface $\varepsilon=20 \% 300 \mathrm{~K}$

(b)
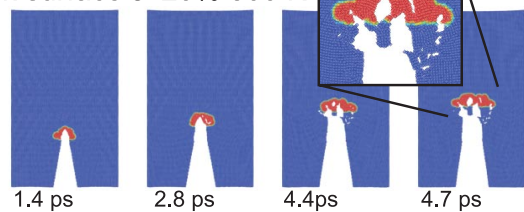

(100) crack surface $\varepsilon=20 \% 300 \mathrm{~K}$
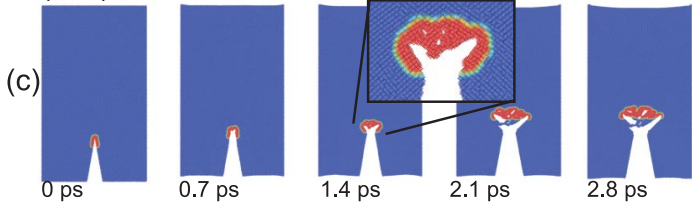

FIG. 4 (color online). (a) Crack dynamics along the [110] direction at finite temperature $(T \approx 300 \mathrm{~K}), 10 \%$ strain applied. (b) The same crack orientation, but $20 \%$ strain applied. (c) Crack dynamics along the [100] direction at finite temperature $(T \approx$ $300 \mathrm{~K}, 10 \%$ strain applied). Shortly after nucleation of the primary crack two branches develop along [110] directions. The systems contain about 64000 atoms and $L_{x} \approx$ $350 \AA \times L_{y} \approx 700 \AA$. 


\section{(a)}

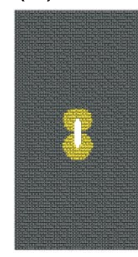

$0 \mathrm{ps}$

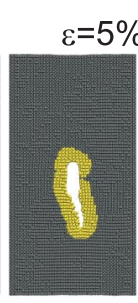

$25 \mathrm{ps}$

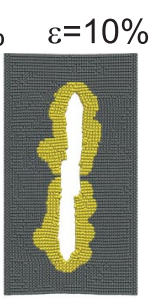

$3.3 \mathrm{ps}$

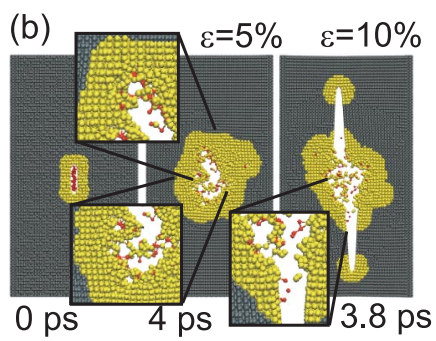

FIG. 5 (color online). Crack dynamics in silicon without (a) and with $\mathrm{O}_{2}$ molecules (b). The gray regions are Tersoff atoms, whereas the colored regions correspond to ReaxFF atoms. The systems contain about 13000 atoms, with $L_{x} \approx 160 \AA \times L_{y} \approx$ $310 \AA$.

Our results underline the importance of large-strain properties at bond breaking for the dynamics of fracture, as suggested earlier by Gao [50] and one of the authors [28,51]. As shown in Fig. 3(c), the Tersoff description leads to a sharp rise of the force close to rupture of bonds, which deviates significantly from the solution by ReaxFF.

Our model is capable of reproducing experimental observations including crack limiting speed, crack instabilities, and directional dependence on crystal orientation. Our studies lead to insights into the atomistic details of the fracture processes, suggesting for example formation of microcracks ahead of a propagating mother crack.

Since ReaxFF is capable of describing a wide heterogeneous range of materials [33-38], our approach may be a practical means to study the coupling of complex chemistry to mechanical properties (Fig. 5). Our hybrid method could enable studies of stress corrosion processes and other degradation and aging mechanisms.

We find that a few thousand atoms in the reactive region at the crack tip are necessary to describe the crack dynamics correctly. Although such calculations are not practical with QM, ReaxFF provides QM accuracy for the reacting part of the system, while retaining speeds comparable to that of simple force fields. The Tersoff potential cannot describe fracture of silicon accurately [10,11], but it is adequate for describing its elastic deformation.

The CMDF development was supported by DARPA and completed with funding from DARPA PROM (C. Schwartz and S. Wax) with support from the Caltech DOE-ASC program. The facilities were supported by DURIP-ONR/ ARO. Development of ReaxFF was supported by NSF-ITR (DMR-0427177) and DARPA-ONR (J. Goldwasser). Approved for Public Release, Distr. Unltd. (DARPA Distr. Stat. "A").

*Corresponding author.

Electronic address: wag@wag.caltech.edu

[1] T. Cramer et al., Phys. Status Solidi A 164, R5 (1997).

[2] D. Holland and M. Marder, Phys. Rev. Lett. 80, 746 (1998).

[3] D. Holland and M. Marder, Adv. Mater. 11, 793 (1999).

[4] J. Hauch et al., Phys. Rev. Lett. 82, 3823 (1999).
[5] F. Ebrahimi and L. Kalwani, Mater. Sci. Eng. A 268, 116 (1999).

[6] T. Cramer et al., Phys. Rev. Lett. 85, 788 (2000).

[7] R. Deegan et al., Phys. Rev. E 67, 066209 (2003).

[8] F. Abraham et al., Comput. Phys. 12, 538 (1998).

[9] F. Abraham et al., MRS Bull. 25, 27 (2000).

[10] J. Swadener et al., Phys. Rev. Lett. 89, 085503 (2002).

[11] N. Bailey and J. Sethna, Phys. Rev. B 68, 205204 (2003).

[12] M. Baskes, Phys. Rev. B 29, 6443 (1984).

[13] A. F. Voter and S.P. Chen, Characterization of Defects (Materials Research Society, Warrendale, PA, 1987), Vol. 82, p. 175.

[14] D. Oh and R. Johnson, J. Mater. Res. 3, 471 (1988).

[15] Y. Mishin et al., Phys. Rev. B 59, 3393 (1999).

[16] R. Komanduri et al., Int. J. Mech. Sci. 43, 2237 (2001).

[17] A. Strachan et al., J. Comput.-Aided Mater. Des. 8, 151 (2001).

[18] B. deCelis et al., J. Appl. Phys. 54, 4864 (1983).

[19] K. Cheung and S. Yip, Model. Simul. Mater., Sci. Eng. 2, 865 (1994).

[20] F. Abraham et al., Phys. Rev. Lett. 73, 272 (1994).

[21] F. Abraham et al., J. Mech. Phys. Solids 45, 1595 (1997).

[22] F. Abraham and H. Gao, Phys. Rev. Lett. 84, 3113 (2000).

[23] H. Gao et al., J. Mech. Phys. Solids 49, 2113 (2001).

[24] F. Abraham et al., Proc. Natl. Acad. Sci. U.S.A. 99, 5783 (2002).

[25] F. Abraham et al., Proc. Natl. Acad. Sci. U.S.A. 99, 5788 (2002).

[26] M. Buehler et al., Comput. Mater. Sci. 28, 385 (2003).

[27] M. Buehler et al., Theor. Appl. Fract. Mech. 41, 21 (2004).

[28] M. Buehler et al., Nature (London) 426, 141 (2003).

[29] M. Buehler et al., Springer Lecture Notes in Computational Science and Engineering, Vol. 39 (Springer, Berlin, 2004), ISBN:3-540-21180-2, p. 143.

[30] J. Tersoff, Phys. Rev. Lett. 61, 2879 (1988).

[31] F. Stillinger and T. Weber, Phys. Rev. B 31, 5262 (1985).

[32] M. Bazant et al., Phys. Rev. B 56, 8542 (1997).

[33] A. van Duin et al., J. Phys. Chem. A 105, 9396 (2001).

[34] A. van Duin et al., J. Phys. Chem. A 107, 3803 (2003).

[35] A. Strachan et al., Phys. Rev. Lett. 91, 098301 (2003).

[36] S. Cheung et al., J. Phys. Chem. A 109, 851 (2005).

[37] K. Nielson et al., J. Phys. Chem. A 109, 493 (2005).

[38] K. Chenoweth et al., J. Am. Chem. Soc. 127, 7192 (2005).

[39] S. Parker et al., IEEE Comput. Sci. Eng. 4, 50 (1997).

[40] http://www.wag.caltech.edu/home/mbuehler/cmdf/.

[41] M. Buehler et al. Combinatorial Methods and Informatics in Materials Science (Materials Research Society, Warrendale, PA, 2006), Vol. 894, p. LL3.3.

[42] D. Beazley et al., Future Gener. Comput. Syst. 19, 599 (2003).

[43] A. Becke, J. Chem. Phys. 98, 5648 (1993).

[44] L. Freund, Dynamic Fracture Mechanics (Cambridge University Press, Cambridge, England, 1990), ISBN:0521-30330-3.

[45] T. Cramer et al., Z. Metallkd. 90, 675 (1999).

[46] J. Fineberg et al., Phys. Rev. Lett. 67, 457 (1991).

[47] J. Fineberg et al., Phys. Rev. B 45, 5146 (1992).

[48] Y. Wu and Y. Xu, Philos. Mag. Lett. 78, 9 (1998).

[49] E. Bouchbinder et al., Phys. Rev. E 70, 046107 (2004).

[50] H. Gao, J. Mech. Phys. Solids 44, 1453 (1996).

[51] M. Buehler and H. Gao, Nature (London) 439, 307 (2006). 